\title{
Endovascular treatment of vertebral artery dissecting aneurysm: A single-center experience
}

\author{
XINTONG ZHAO ${ }^{1}$, HUIFANG WANG ${ }^{2}$, JIAQIAING LIU ${ }^{1}$, ZIHUAN ZHANG ${ }^{1}$ and ZHENBAO LI ${ }^{1}$ \\ Departments of ${ }^{1}$ Neurosurgery and ${ }^{2}$ Pharmacy, Yijishan Hospital, Wannan Medical College, Wuhu, Anhui 241001, P.R. China
}

Received April 20, 2019; Accepted September 20, 2019

DOI: 10.3892/etm.2019.8128

\begin{abstract}
The aim of the present study was to explore the optimal treatment strategy for patients with vertebral artery dissecting aneurysms via the endovascular approach. A total of 47 patients (50 aneurysms, including 28 ruptured and 22 unruptured) underwent endovascular treatment between January 2012 and March 2018. Of these aneurysms, 18 were treated by parent artery occlusion, 11 by stent implantation and 21 by stent-assisted coiling. The complete occlusion rate was $100 \%(18 / 18)$ in aneurysms treated by parent artery occlusion and $46.9 \%(15 / 32)$ for stent implantation with or without coiling. External drainage was performed in 8 patients with ruptured aneurysms. Post-operative bleeding occurred in 2 patients treated by stent-assisted coiling. Ventriculo-peritoneal shunt was performed in 4 patients due to delayed hydrocephalus. Post-operative frontal hematoma occurred in one patient treated by stent-assisted coiling. The mortality was $6.4 \%$ (3/47) prior to discharge. The recurrence rate was $8.3 \%(1 / 12)$ in aneurysms treated by parent artery occlusion and $13.6 \%(3 / 22)$ in those treated by stent implantation with or without coiling. A total of 39 patients were followed up for $14.56 \pm 14.91$ months. The good outcome rates were $100 \%$ (28/28) in patients with unruptured aneurysms and a Hunt-Hess grade of $1-3$, and $72.7 \%(8 / 11)$ in patients with a Hunt-Hess grade of 4 or 5 . In conclusion, the present study indicated that endovascular treatment is a safe and efficient method for patients with vertebral artery aneurysm. The treatment strategy should be based on the patient's condition and aneurysmal characteristics. Parent artery occlusion may be
\end{abstract}

Correspondence to: Dr Zhenbao Li, Department of Neurosurgery, Yijishan Hospital, Wannan Medical College, 2 Zheshan Road, Wuhu, Anhui 241001, P.R. China

E-mail: zhenbaoli456@126.com

Abbreviations: VADA, vertebral artery dissecting aneurysm; DSA, digital subtraction angiography; PICA, posterior inferior cerebellar artery; GOS, Glasgow Outcome Scale

Key words: vertebral artery dissecting aneurysm, endovascular treatment, morbidity, follow-up, outcomes suitable for selected patients with ruptured vertebral artery aneurysms.

\section{Introduction}

Vertebral artery dissecting aneurysm (VADA) is a rare condition accounting for $3 \%$ of intracranial aneurysms. VADA may result in subarachnoid hemorrhage and ischemic stroke (1). However, the incidence may be underestimated due to a high rate of mortality during the early stages as a result of mortality from severe hemorrhaging. It is necessary to treat a ruptured VADA as soon as possible due to the high risk of rebleeding and mortality. Craniotomy remains a challenge for neurosurgeons. Endovascular treatment is a suitable alternative option for treating patients with VADA. It is difficult to treat VADA with a coil, and additional assistive technologies are required. The stent-assisted coiling technique extends the usage of endovascular treatment to VADA. Endovascular treatment includes destruction (parent artery occlusion) and reconstruction techniques (stent implantation with or without coiling) (2-5). However, there is currently controversy regarding the preferred treatment option. Therefore, it is necessary to determine whether endovascular treatment results in favorable outcomes in patients with VADA, and which endovascular modalities is optimal for each specific subtype of VADA. In the present study, data from 47 patients with 50 VADAs were collected and the clinical and angiographic outcomes were analyzed.

\section{Materials and methods}

Patient population. A total of 47 patients with 50 VADAs undergoing endovascular treatment between January 2012 and March 2018 at Yijishan Hospital, Wannan Medical College (Wuhu, China) were included in the present study. Following approval from the institutional review board, the clinical and radiological materials were collected. The inclusion criterion was that VADAs were confirmed by digital subtraction angiography (DSA). The exclusion criterion was that VADAs originated from the extracranial vessel or involved the basilar artery. Hunt-Hess grading was used to evaluate the condition of the patients on admission. The clinical data are presented in Table I. The cohort was comprised of 31 males and 16 females with a mean age of $54.57 \pm 10.27$ years (age range, $28-75$ years). A total of 22 patients $(46.8 \%)$ presented with hypertension, 28 patients were admitted to hospital due to subarachnoid 
hemorrhage, 14 had ischemic events and 5 had other irrelevant presentations. In addition, 15 patients were admitted to hospital with a Hunt-Hess grade of 4 or 5 and 13 with a Hunt-Hess grade of $1-3$.

Radiological characteristics. Subarachnoid hemorrhage was confirmed by CT scanning. All VADAs were confirmed by DSA with or without CTA or MRA. They were defined as dominant vertebral arteries when there was no contralateral vertebral artery or the ipsilateral vertebral artery was significantly larger compared with the contralateral one.

Among the 47 cases, three patients had bilateral VADAs. The radiological characteristics of the aneurysms are presented in Table II. There were 28 ruptured aneurysms and 22 unruptured ones; furthermore, 25 aneurysms were located on the left vertebral artery and 25 on the right one. A total of 13 aneurysms were located at the dominant vertebral artery and 37 at the non-dominant vertebral artery. According to the association between the aneurysms and the posterior inferior cerebellar artery (PICA), the aneurysms were divided into three types: Proximal, distal and with involvement of the PICA. Based on this classification, 11 aneurysms were proximal to the PICA, 27 were distal to the PICA and 12 aneurysms had involvement with the PICA.

Treatment. The anti-platelet drugs (aspirin $100 \mathrm{mg} / \mathrm{day}$ and clopidogrel $75 \mathrm{mg} / \mathrm{day}$ ) were administered for 3 days prior to the procedure for patients with unruptured aneurysms. All patients with ruptured aneurysms received endovascular treatment within $24 \mathrm{~h}$ to reduce the risk of rebleeding, except one patient who initially refused endovascular treatment. A loading dose of anti-platelet drugs (aspirin and clopidogrel, $300 \mathrm{mg}$ each) was administered to patients with ruptured aneurysms who were to undergo stent deployment by oral or nasal feeding. The anti-platelet drugs (aspirin $100 \mathrm{mg} /$ day and clopidogrel $75 \mathrm{mg} /$ day) were administered for 6 weeks post-operatively, followed by aspirin alone.

The treatment strategy was formulated by experts according to the patients' clinical presentation, whether the aneurysm had ruptured, involvement of the dominant or non-dominant vertebral artery, and association between the aneurysms and the PICA. Parent artery occlusion was performed when the vertebral artery involved was non-dominant and the PICA was not involved. Stent implantation with or without coiling was performed in patients with unruptured aneurysms and in patients with ruptured aneurysms when the PICA was involved or the aneurysm was located on the dominant vertebral artery.

All procedures were performed under general anesthesia. A $6 \mathrm{~F}$ sheath was placed into the right femoral artery and a $6 \mathrm{~F}$ guiding catheter was placed into the ipsilateral vertebral artery. In certain instances, a $5 \mathrm{~F}$ or $6 \mathrm{~F}$ sheath was placed into the bilateral femoral artery when a bilateral vertebral artery approach was required. The stent-delivery-catheter was navigated into the basilar artery on the roadmap. A suitable stent was selected according to the length of the vessel involved. The stent should at least cover the proximal and distal part of a lesioned vessel. The diameter of a stent should be larger than that of the parent artery in order to obtain stability and adherence to the vessel. An enterprise or LVIS stent was used to reconstruct the parent artery. For patients undergoing parent artery occlusion, two micro-catheters were placed into the lesioned area in order to obtain dense embolization. For patients with bilateral aneurysms, the responsible aneurysm was estimated according to the bleeding characteristics and aneurysmal figuration. The responsible aneurysm was first treated and the other aneurysm was treated at a second stage.

Follow-up. Follow-up by DSA was scheduled to be performed 3 months after the procedure, as well as 9 and 21 months after the operation. The immediate angiographic outcomes were divided into complete occlusion and incomplete occlusion. The angiographic outcomes at follow-up were classified as follows: Complete regression (no contrast filling), incomplete regression (no change in coil configuration, obliteration grade or contrast filling) and recurrence (aneurysm recurrence evident due to aneurysm growth or coil compaction). The clinical outcomes were evaluated by using the Glasgow Outcome Scale (GOS). Favorable outcomes were defined as GOS 4 or 5 and poor outcomes were defined as GOS 1-3.

Statistical analysis. Statistical analysis was performed using SPSS for Windows v.17.0 (IBM Corp.). The Chi-squared test was used to compare the occlusion rate between parent artery occlusion and stent implantation with or without coiling. The favorable outcome rate between ruptured aneurysms and unruptured aneurysms was also compared using the chi-squared test. $\mathrm{P}<0.05$ was considered to indicate a statistically significant difference.

\section{Results}

Treatment and peri-operative morbidities. The aneurysms were treated as follows: 18 were treated by parent artery occlusion, 11 by stent implantation (1 aneurysm by single LVIS, 7 by double enterprise and 3 by triple enterprise) and 21 by stent-assisted coiling ( 7 aneurysms by single enterprise, 12 by double enterprise and 2 by triple enterprise). There were three patients with bilateral VADAs. Of these three patients, one patient with unruptured bilateral VADAs underwent double stent implantation in stages. The second patient with ischemic stroke underwent stent-assisted coiling and another aneurysm was treated by parent artery occlusion at the second stage. For the third patient with subarachnoid hemorrhage, the major aneurysm was subjected to stent-assisted coiling and another aneurysm underwent stent-assisted coiling during the second stage. Complete occlusion was achieved in 33 aneurysms and incomplete occlusion in 17 aneurysms. All aneurysms treated by parent artery occlusion gained complete occlusion. Complete occlusion was achieved in $46.9 \%$ (15/32) of aneurysms by stent implantation with or without coiling. There was a significant difference in the complete occlusion rate between aneurysms treated by parent artery occlusion and stent implantation with or without coiling $(\mathrm{P}<0.001)$.

There was no evidence of intra-operative rupture in any of the procedures. Intra-operative thrombosis occurred in one patient treated by stent-assisted coiling; this patient developed symptoms of cerebellar infarction. No ischemic symptoms were observed in the patients treated by parent artery occlusion. External ventricular drainage was performed in 8 patients with ruptured aneurysms. Post-operative bleeding occurred in 
Table I. Characteristics of the patients with vertebral artery dissecting aneurysms $(n=47)$.

\begin{tabular}{lc}
\hline Characteristics & $\mathrm{N}(\%)$ \\
\hline Gender & \\
Male & 31 \\
Female & 16 \\
Age (years) & \\
Mean & $54.57 \pm 10.27$ \\
Median & 54 \\
Range & $28-75$ \\
Hypertension & $22(46.8)$ \\
Hunt-Hess grading & \\
1-3 & $13(27.7)$ \\
4-5 & $15(31.9)$ \\
Initial presentation & \\
SAH & $28(59.6)$ \\
Ischemic events & $14(29.8)$ \\
Other & $5(10.6)$ \\
\hline
\end{tabular}

Values are expressed as n unless otherwise specified. SAH, subarachnoid hemorrhage.

Table II. Radiological characteristics of aneurysms.

\begin{tabular}{lc}
\hline Characteristics & $\mathrm{N}(\%)$ \\
\hline Total & 50 \\
Ruptured & $28(56)$ \\
Unruptured & $22(44)$ \\
Left/right & $25 / 25$ \\
Blood supply to basilar artery & \\
Dominant & $13(26)$ \\
Non-dominant & $37(74)$ \\
Location & \\
Proximal & $11(22)$ \\
Distal & $27(54)$ \\
Involvement & $12(24)$ \\
\hline
\end{tabular}

two patients treated by stent-assisted coiling. Post-operative frontal hematoma occurred in one patient due to a puncture. Another patient received craniotomy due to subdural hematoma. No post-operative bleeding occurred in patients treated by parent artery occlusion. Peri-operative morbidity occurred in $10.7 \%(3 / 28)$ of patients with a ruptured aneurysm. There was no peri-operative morbidity in patients with unruptured aneurysms (Table III).

Ventriculo-peritoneal shunt was performed in 4 patients due to delayed hydrocephalus. Post-operative frontal hematoma occurred in one patient treated by stent-assisted coiling three days after ventriculo-peritoneal shunt, which caused an obstruction of the shunt (Fig. 1). Ventriculo-peritoneal shunt was performed at the contralateral side.
Table III. Treatment and morbidities.

\begin{tabular}{lr}
\hline Treatment/outcomes & $\mathrm{N}(\%)$ \\
\hline Treatment & $18(36)$ \\
Parent artery occlusion & $11(22)$ \\
Stent implantation & $21(42)$ \\
Stent-assisted coiling & $33(66)$ \\
Outcomes & $17(34)$ \\
Complete occlusion & $18 / 18(100)^{\mathrm{a}}$ \\
Incomplete occlusion & $15 / 32(46.9)^{\mathrm{a}}$ \\
Complete occlusion rate after parent & \\
artery occlusion & $3 / 28(10.7)$ \\
Complete occlusion rate after stent & \\
placement & $0(0)$ \\
Perioperative morbidity for ruptured & \\
aneurysms & \\
Perioperative morbidity for unruptured & \\
aneurysms &
\end{tabular}

${ }^{a}$ The complete occlusion rate after parent artery occlusion was significantly higher than that after stent placement $(\mathrm{P}<0.001)$. Values are expressed as $n$ unless otherwise specified.

Angiographic outcomes. In total, 31 patients (34 aneurysms) were followed up for 11.97 \pm 12.96 months (range, 3-52 months) by DSA. Of these, 4 aneurysms in 4 patients $(11.8 \%)$ presented with recurrence. The recurrence rate was $8.3 \%(1 / 12)$ in aneurysms treated by parent artery occlusion (Fig. 2) and $13.6 \%(3 / 22)$ in aneurysms treated by stent implantation with or without coiling (Table IV). In 6 patients initially treated by stent implantation, four aneurysms obtained incomplete regression and two achieved complete occlusion (Fig. 3). Recurrence occurred in 3 of the 16 aneurysms treated by stent-assisted coiling.

Clinical outcomes. The mortality rate was $6.4 \%(3 / 47)$ in the patients prior to discharge (Table IV). These deaths all occurred in patients with a Hunt-Hess grade of 5 on admission. The outcome at discharge was GOS grade 2 in 2 patients, GOS grade 3 in 5 patients, GOS grade 4 in 4 patients and GOS grade 5 in 33 patients. Favorable outcomes were achieved in $78.7 \%(37 / 47)$ of the entire cohort. Among the 15 patients with a Hunt-Hess grade of 4 or 5 on admission, the outcome at discharge was GOS grade 1 in 3 patients, GOS grade 2 in 2 patients, GOS grade 3 in 5 patients, GOS grade 4 in 2 patients and GOS grade 5 in 3 patients. Favorable outcomes were achieved in $64.3 \%(18 / 28)$ of the patients with ruptured aneurysms on admission. All 19 patients with unruptured aneurysms on admission obtained favorable outcomes. There was a significant difference in favorable outcomes between patients with ruptured aneurysms and unruptured aneurysms $(\mathrm{P}=0.003)$.

A total of 3 patients had died prior to discharge and 5 patients $(10.6 \%)$ were lost to follow-up; the remaining 39 patients were followed up for 14.56 \pm 14.91 months (range, 3-63 months) by telephone and second admission. GOS grade 3 was obtained 

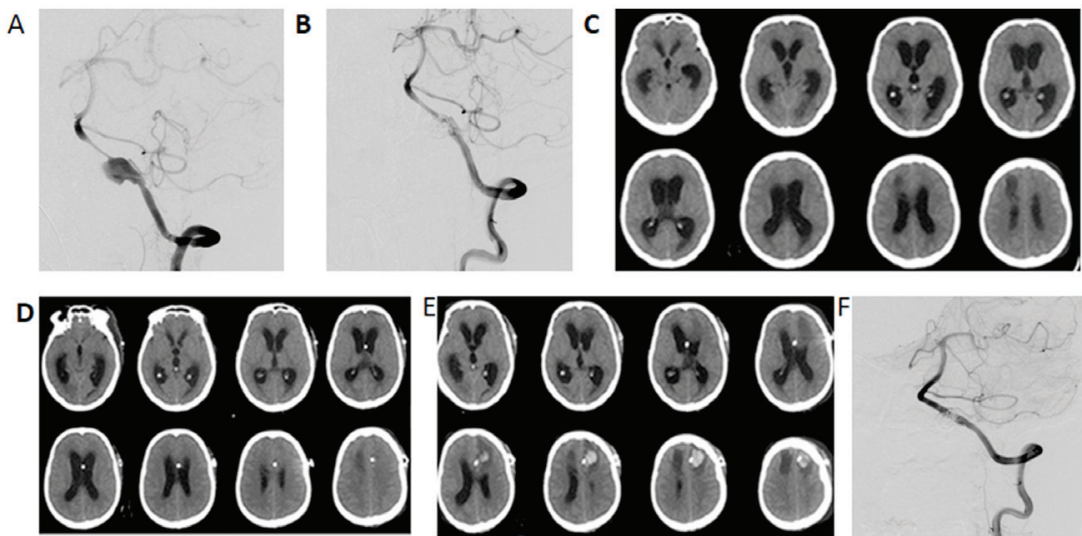

Figure 1. Images of a patient with left ruptured VADA, treated with stent-assisted coiling immediately on admission. (A) Emergency angiography indicated VADA and posterior inferior cerebellar artery originating from the dissecting aneurysm. (B) Angiography image taken immediately after the procedure. (C) CT scan of the hydrocephalus three weeks after the procedure. (D) CT scan on the first day after ventriculo-peritoneal shunt. (E) CT scanning indicating the shunting was obstructed due to frontal hematoma three days after ventriculo-peritoneal shunt. (F) Angiographic follow-up indicated that the aneurysm was completely occluded eight months after endovascular treatment. VADA, vertebral artery dissecting aneurysm.
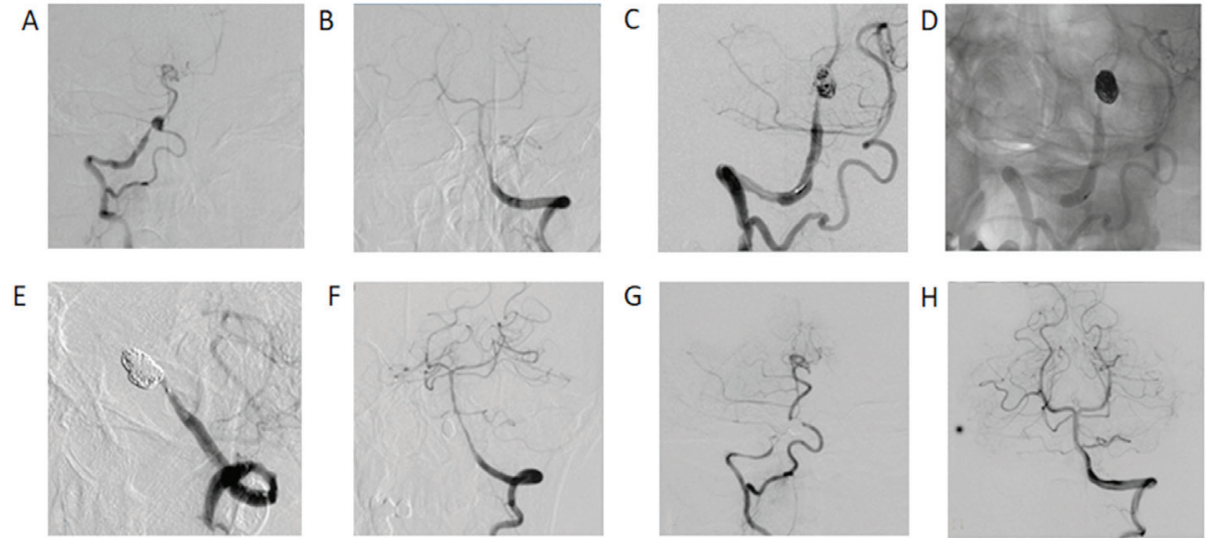

Figure 2. Images of a patient with right-ruptured VADA, treated with parent artery occlusion immediately on admission. (A) Emergency angiography revealed right VADA and posterior inferior cerebellar artery originating from the proximal segment of the vertebral artery. (B) Image of the left vertebral artery. (C) VADA was treated by parent artery occlusion. (D) The process of coiling. (E) Aneurysm and the right vertebral artery were completely occluded. (F) Image of the left vertebral artery after the procedure. (G) Angiographic follow-up of the right vertebral artery indicated that there was no recurrence five months after the procedure. $(\mathrm{H})$ Images of the left vertebral artery at follow-up. VADA, vertebral artery dissecting aneurysm.
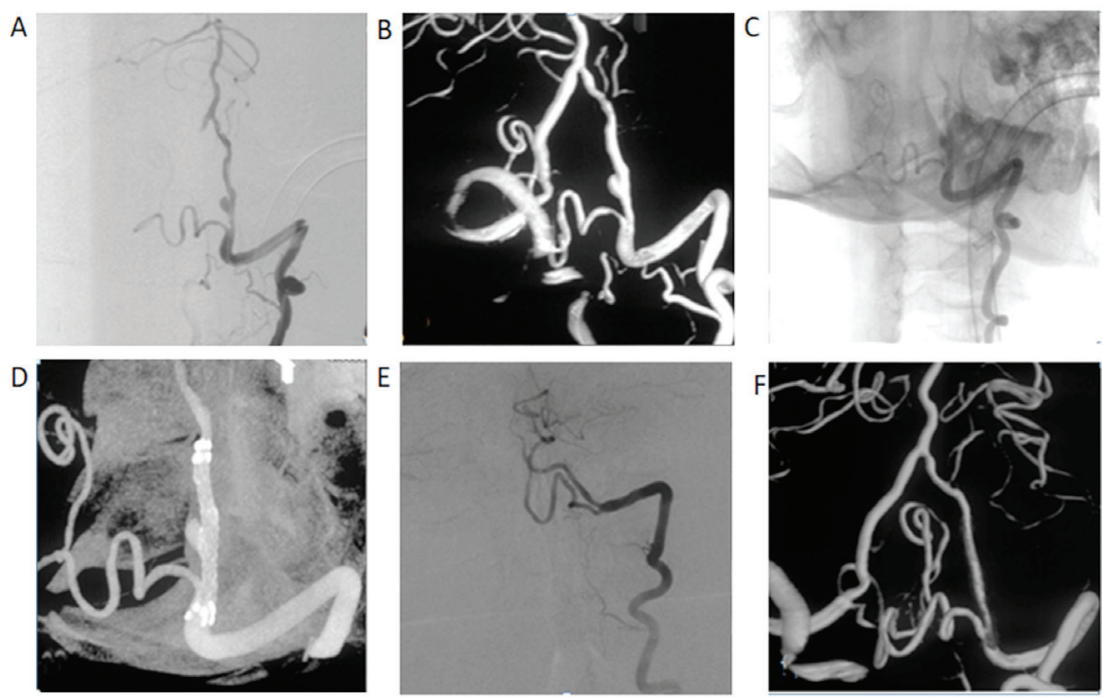

Figure 3. Images of a patient with left unruptured VADA, which was electively treated with double-stent implantation. (A) Images of the vertebral artery indicated the VADA prior to the procedure. (B) Three-dimensional image of the aneurysm. (C) Image after double-stent implantation. (D) Dynamic CT angiography after the procedure. (E) Angiographic follow-up indicated that the aneurysm was completely occluded four months after the procedure. (F) Three-dimensional image of the vertebral artery. VADA, vertebral artery dissecting aneurysm. 
Table IV. Angiographic and clinical outcomes.

\begin{tabular}{lc}
\hline Outcomes & $\mathrm{N}(\%)$ \\
\hline Angiographic outcomes & \\
Recurrence rate after parent artery occlusion & $1 / 12(8.3)$ \\
Recurrence rate after stent & $3 / 22(13.6)$ \\
Clinical outcomes at discharge & \\
GOS 1 & $3 / 47(6.4)$ \\
GOS 2 & $2 / 47(4.3)$ \\
GOS 3 & $5 / 47(10.6)$ \\
GOS 4 & $4 / 47(8.5)$ \\
GOS 5 & $33 / 47(70.2)$ \\
Favorable outcomes for ruptured aneurysms & $18 / 28(64.3)^{\mathrm{a}}$ \\
Favorable outcomes for unruptured aneurysms & $19 / 19(100)^{\mathrm{a}}$ \\
Clinical outcomes at follow-up & \\
GOS 3 & $3(7.7)$ \\
GOS 4 & $3(7.7)$ \\
GOS 5 & $33(84.6)$ \\
\hline
\end{tabular}

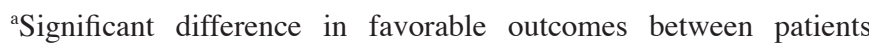
with ruptured aneurysms and unruptured aneurysms $(\mathrm{P}=0.003)$. GOS, Glasgow Outcome Scale.

in 3 patients, GOS grade 4 in 3 patients and GOS grade 5 in 33 patients at the end of the follow-up. Favorable outcomes were achieved in $92.3 \%$ (36/39) of the patients. The favorable outcome rate was $72.7 \%(8 / 11)$ in patients with a Hunt-Hess grade of 4 or 5 on admission and $100 \%$ (28/28) inpatients with unruptured aneurysms and a Hunt-Hess grade of 1-3 on admission.

\section{Discussion}

VADA usually occurs in adult patients aged 40-50 years (1). The major manifestations are subarachnoid hemorrhage and ischemic events. The diagnosis is based on CTA, MRA or DSA. DSA is currently the golden standard for the diagnosis of VADA. The rate of rebleeding and mortality is high in patients with ruptured VADA (6-9). Yamada et al (7) reported on 24 conservatively treated patients with ruptured VADA. Recurrent hemorrhage occurred in $58 \%$ of the patients and $46 \%$ of those cases died due to massive rebleeding. Timely treatment may reduce the rate of mortality and morbidity resulting from rebleeding. Therefore, the majority of the patients in the present study underwent endovascular treatment during the first $24 \mathrm{~h}$. The treatment for patients with an unruptured VADA remains controversial. According to certain clinicians, conservative treatment may achieve good outcomes (9). Guan et al (10) suggested that patients with enlarged VADA aneurysms and those with progressive ischemia following medical treatment should be treated using an endovascular approach. Naito et al (11) indicated that patients with VADA with relatively large or growing dilations should receive endovascular treatment. An unruptured VADA should be treated due to a higher mortality rate following rupture, particularly for aneurysms with irregular figuration or morphological changes, multiple aneurysms or when combined with hypertension.

The primary treatment strategy consists of surgery and endovascular embolization. Ligation of the lesioned artery and blood reconstruction may be performed by surgery (vertebral artery or occipital artery-PICA bypass) $(12,13)$. However, it may be difficult to perform surgical treatment due to a deep location, adjacent brain stem and posterior cranial nerves. Endovascular embolization is the preferred strategy for VADA due to its safety and efficiency, which includes reconstruction and destruction techniques $(1,14)$. Destruction technique refers to parent artery occlusion. The reconstruction technique consists of stent implantation with or without coiling and flow-diverting stent.

The destruction technique is the most effective method to prevent aneurysm rupture and rebleeding $(2,3)$. The immediate obliteration rate is high. The obliteration rate was $100 \%$ in the patients included in the present study, which was similar to that in other studies. Madaelil et al (3) reported on 12 patients with VADA involving distal vertebral artery $(n=10)$ or PICA $(n=2)$ treated by parent artery occlusion. Only one patient suffered from ischemic stroke and one patient died prior to discharge. There was no recurrence at follow-up and 10 patients $(83 \%)$ achieved favorable outcomes. A meta-analysis by Guan et al (10) demonstrated that the immediate obliteration rate was higher in patients treated with parent artery occlusion compared with that in patients treated with stent-assisted coiling. However, there was no significant difference in clinical outcomes and recurrence rate between them in the long-term follow-up. The efficacy of parent artery occlusion comes at the expense of a lesioned vertebral artery. Parent artery occlusion may result in severe complications when the lesioned artery is dominant compared with the contralateral side or if the PICA is involved. Parent artery occlusion should be performed when the basilar artery and the PICA are able to maintain a sufficient blood supply (15). There was no evidence of brain infarction in the 18 patients treated by parent artery occlusion in the present study. The rate of favorable outcome for patients with ruptured aneurysms in the present study was $64.3 \%$, which appeared to be worse than that reported in previous studies $(5,16)$. However, this may be due to high Hunt-Hess grades in the cohort of the present study. Patients with a Hunt-Hess grade of 4 or 5 accounted for $53.6 \%(15 / 28)$ of cases with ruptured aneurysms.

The aim of stent implantation with or without coiling is to keep the vertebral artery unobstructed and reduce the pressure of blood flow on the vessel wall by maintaining the flow direction. The coil facilitates the repair of dissection by thrombosis. Multiple stents may increase the metal coverage. Zhao et al (5) reported on 57 patients with VADA treated by stent-assisted coiling. The peri-operative morbidity rate was $5 \%(3 / 57)$ and the overall mortality rate was 5\% (3/57). After an average of 27 months of angiographic follow-up, 42 of the 54 patients obtained complete occlusion, 7 exhibited improved results and 5 experienced recurrence. They indicated that immediate incomplete occlusion was an independent risk factor for recurrence. After a mean follow-up of 62 months, 47 patients obtained favorable outcomes (mRS, 0-1), and high age $(56.90 \pm 10.88$ years) and worse Hun-Hess grading were associated with poor outcome (5). 
Chung et al (4) reported on 8 patients with 9 VADAs (including 1 ruptured and 8 unruptured) treated with triple stent implantation. A total of 8 unruptured VADAs returned to normal after 3 months of angiographic follow-up and 1 patient suffered from recurrence and received double stent implantation. Finally, 7 patients obtained favorable outcomes after a median clinical follow-up of 22.6 months. Flow-diversion, including pipeline embolization device, has higher metal coverage. An increasing number of studies have been reporting on the use of flow-diversion in the treatment of VADA. Narata et al (15) suggested that flow-diversion modifies aneurysmal hemodynamics and accelerates thrombosis.

However, the use of stents may increase the risk of an ischemic event during the acute phase (16). The use of anti-platelet drugs may increase the rate of rebleeding, particularly in patients with an incomplete occlusion. It may result in post-operative hematoma due to puncture, particularly in patients with acute hydrocephalus requiring external drainage. In the present study, 8 patients received external ventricular drainage. Although heparin was neutralized by protamine, one patient exhibited subdural hematoma and another patient suffered from frontal hematoma due to puncture. All hematoma occurred in patients treated with stent-assisted coiling. Ventriculo-peritoneal shunt was performed in 4 patients due to delayed hydrocephalus. Post-operative frontal hematoma occurred in one patient treated with stent-assisted coiling three days after shunting. Ventriculo-peritoneal shunt was performed on the contralateral side.

There are certain limitations to the present study. It was a retrospective and non-randomized study. The number of patients and duration of follow-up were also limited. Certain patients were lost to follow-up due to unfavorable outcomes or loss of contact information.

In conclusion, endovascular treatment of VADA is an effective and safe method. Stent implantation with or without coiling is suitable for unruptured VADA, PICA involvement and dominant vertebral artery. In cases with a ruptured VADA, parent artery occlusion may be preferred, as the obliteration rate is higher. It may decrease the probability of post-operative rebleeding and reduce the requirement for anti-platelet drugs, which increases the probability of post-operative rebleeding, particularly in patients with acute hydrocephalus requiring external drainage.

\section{Acknowledgements}

Not applicable.

\section{Funding}

The present study was supported by the National Natural Science Foundation of China (grant no. 81572486).

\section{Availability of data and materials}

The datasets used and/or analyzed during the current study are available from the corresponding author on reasonable request.

\section{Authors' contributions}

$\mathrm{XZ}$ was involved in drafting the manuscript, revising it critically for important intellectual content and analysing the data; HW made substantial contributions to acquisition of data, or analysis and interpretation of data; JL made substantial contributions to conception, design and analysis. $\mathrm{ZZ}$ made substantial contributions to analysis and revising it critically.

\section{Ethics approval and consent to participate}

The study was approved by the ethics committee of Yijishan Hospital, Wanan Medical College (Wuhu, China). Written informed consent was obtained from the patients or their guardians.

\section{Patient consent for publication}

Not applicable.

\section{Competing interests}

The authors declare that they have no competing interests.

\section{References}

1. Su W, Gou S, Ni S, Li G, Liu Y, Zhu S and Li X: Management of ruptured and unruptured intracranial vertebral artery dissecting aneurysms. J Clin Neurosci 18: 1639-1644, 2011.

2. Urasyanandana K, Withayasuk P, Songsaeng D, Aurboonyawat T, Chankaew E and Churojana A: Ruptured intracranial vertebral artery dissecting aneurysms: An evaluation of prognostic factors of treatment outcome. Interv Neuroradio 23: 240-248, 2017.

3. Madaelil TP, Wallace AN, Chatterjee AN, Zipfel GJ, Dacey RG Jr, Cross DT III, Moran CJ and Derdeyn CP: Endovascular parent vessel sacrifice in ruptured dissecting vertebral and posterior inferior cerebellar artery aneurysms: Clinical outcomes and review of the literature. J Neurointerv Surg 8: 796-801, 2016.

4. Chung Y, Lee SH, Choi SK, Kim BJ, Lee KM and Kim EJ: Triple stent therapy for the treatment of vertebral dissecting aneurysms: Efficacy and safety. World Neurosurg 99: 79-88, 2017.

5. Zhao KJ, Fang YB, Huang QH, Xu Y, Hong B, Li Q, Liu JM, Zhao WY and Deng BQ: Reconstructive treatment of ruptured intracranial spontaneous vertebral artery dissection aneurysms: Long-term results and predictors of unfavorable outcomes. PLoS One 8: e67169, 2013.

6. Jin SC, Kwon DH, Choi CG, Ahn JS and Kwun BD: Endovascular strategies for vertebrobasilar dissecting aneurysms. AJNR Am J Neuroradiol 30: 1518-1523, 2009.

7. Yamada I, Kitahara T, Kurata A, Fujii K and Miyasaka Y: Intracranial vertebral artery dissection with subarachnoid hemorrhage: Clinical characteristics and outcomes in conservatively treated patients. J Neurosurg 101: 25-30, 2004.

8. Mizutani T, Aruga T, Kirino T, Miki Y, Saito I and Tsuchida T: Recurrent subarachnoid hemorrhage from untreated ruptured vertebrobasilar dissecting aneurysms. Neurosurgery 36: 905-923, 1995.

9. Arnold M, Bousser M, Fahrni G, Fischer U, Georgiadis D, Gandjour J, Benninger D, Sturzenegger M, Mattle HP and Baumgartner RW: Vertebral artery dissection: Presenting findings and predictors of outcome. Stroke 37: 2499-2503, 2006.

10. Guan J, Li G, Kong X, He C, Long J, Qin H, Zhang H and Wang R: Endovascular treatment for ruptured and unruptured vertebral artery dissecting aneurysms: A meta-analysis. J Neurointerv Surg 9: 558-563, 2017.

11. Naito I, Iwai T and Sasaki T: Management of intracranial vertebral artery dissections initially presenting without subarachnoid hemorrhage. Neurosurgery 51: 937-938, 2002. 
12. Czabanka M, Ali M, Schmiedek P, Vajkoczy P and Lawton MT: Vertebral artery-posterior inferior cerebellar artery bypass using a radial artery graft for hemorrhagic dissecting vertebral artery aneurysms: Surgical technique and report of 2 cases. J Neurosurg 114: 1074-1079, 2011.

13. Park W, Ahn JS, Park JC, Kwun BD and Kim CJ: Occipital artery-posterior inferior cerebellar artery bypass for the treatment of aneurysms arising from the vertebral artery and its branches. World Neurosurg 82: 714-721, 2014.

14. Han J, Lim DJ, Ha SK, Choi JI, Jin SW and Kim CJ: Endovascular treatment of symptomatic vertebral artery dissecting aneurysms. J Cerebrovasc Endovasc Neurosurg 18: 201-207, 2016
15. Narata AP, Yilmaz H, Schaller K, Lovblad KO and Pereira VM: Flow-diverting stent for ruptured intracranial dissecting aneurysm of vertebral artery. Neurosurgery 70: 982-989, 2012.

16. Bechan RS, Sprengers ME, Majoie CB, Peluso JP, Sluzewski M and van Rooij WJ: Stent-Assisted coil embolization of intracranial aneurysms: Complications in acutely ruptured versus unruptured aneurysms. AJNR Am J Neuroradiol 37: 502-507, 2016.

This work is licensed under a Creative Commons Attribution-NonCommercial-NoDerivatives 4.0 International (CC BY-NC-ND 4.0) License. 